\title{
Average Error Rates and Achievable Capacity in Large Office Indoor Wireless Environments
}

\author{
Indrakshi Dey, Member, IEEE, Geoffrey G. Messier, Member, IEEE, and Sebastian Magierowski, Member, IEEE
}

\begin{abstract}
Performance of common digital modulation techniques is analyzed over indoor wireless environments modeled through the recently proposed joint fading and two-path shadowing (JFTS) channel model. Mathematically tractable expressions for the instantaneous signal-to-noise ratio statistics, average bit error rates, and achievable channel cutoff rates are derived. Analytical results are used to: 1 ) investigate the impact of different JFTS model parameters and different modulation techniques on bit error rates and cutoff rates and 2) demonstrate how the JFTS channel model affects system performance in comparison with conventional empirical channel models. Finally simulation results are used to corroborate this analysis and evaluate the usefulness of such an analysis.
\end{abstract}

Index Terms-JFTS, ABER, $M$-QAM, $M$-PSK.

\section{INTRODUCTION}

\section{A. Motivation}

$\mathbf{T}$ THE wide variety of applications of indoor wireless communication has resulted in the increased demand of exact theoretical analysis of high capacity wireless communication systems when implemented on indoor wireless access points. For example, expressions for the average bit error rate (ABER) are essential for designing effective signaling and error control coding schemes, as they provide insights into how system parameters affect performance and are more computationally efficient for analyzing system performance.

It is well-known that indoor wireless links are affected by both small scale fading and shadowing effects. Therefore, it will be more accurate to account for the combined small and large scale channel effects while evaluating system performance. Common composite channel models [1], [2], [3] that combine large and small scale channel effects have been developed primarily for outdoor channels, where the users are highly mobile transiting through several scattering clusters. As a result, a range of main waves arrive at the mobile, the strength of each of which can be drawn from the log-normal or the Gamma distribution.

In an indoor wireless environment, the path between the access point and the users is too short for shadowing to be

Manuscript received December 21, 2016; revised April 30, 2017 and June 18, 2017; accepted July 12, 2017. The associate editor coordinating the review of this paper and approving it for publication was J. Choi. (Corresponding author: Indrakshi Dey.)

I. Dey and G. G. Messier are with the Department of Electrical and Computer Engineering, University of Calgary, Calgary, AB T2N 1N4, Canada (e-mail: deyi@ucalgary.ca; gmessier@ucalgary.ca).

S. Magierowski is with the Department of Electrical Engineering and Computer Science, Lassonde School of Engineering, York University, Toronto, ON M3J 1P3, Canada (e-mail: magiero@cse.yorku.ca).

Digital Object Identifier 10.1109/TCOMM.2017.2729552 accurately characterized by the log-normal distribution and the mobile users restrict their movement within a small area due to the incapability of most WLAN standards to handle hand-offs efficiently. A new composite channel model, called the joint fading and two-path shadowing (JFTS) model, is proposed in [4]. Based on an extensive channel measurement campaign, the JFTS model is shown to be a more accurate model for characterizing indoor WLAN channels.

The JFTS distribution has also some added advantages over other common composite channel models. Since the JFTS distribution is a general model that includes a wide variety of channel conditions as special cases (no fading, no shadowing, deep fading, heavy shadowing, etc.), the expressions for different performance metrics evaluated over the JFTS channel will provide us with the achievable performance measures over a large variety of practical channel conditions.

The JFTS distribution is fundamentally different from other composite fading/shadowing models. It is a convolution of the Ricean fading and the two-wave with diffused power (TWDP) shadowing models [5]. It follows the bivariate non-centralized chi-squared distribution and therefore, cannot approach Gaussian statistics. This is unlike conventional fading models that can approach zero-mean complex Gaussian statistics under different propagation conditions. This suggests that performance of different communication systems over the JFTS channel will be very different than what has been predicted by conventional fading/shadowing models and motivates the need for analytical error rate and achievable capacity expressions specifically for the JFTS channel.

The work in [4] is extended in [6] and [7] by deriving expressions for the first-order statistics and Cumulative Distribution Function (CDF) of the JFTS distribution respectively. In [8], expressions for ergodic capacity achievable by different adaptive transmission techniques over a JFTS channel are derived. The effect of these adaptive schemes on the relationship between optimal cutoff signal-to-noise ratio (SNR) and the average received SNR for JFTS faded/shadowed links is also studied in [8]. However, to the best of the authors' knowledge, a detailed study on how different $M$-ary coherent and noncoherent modulation techniques perform when applied to a JFTS faded/shadowed link has not yet been conducted in literature.

\section{B. Main Results and Paper Organization}

The main contributions of the paper are summarized as follows. 
- We derive analytically tractable expressions for ABER of coherent and noncoherent binary, and coherent $M$-ary modulation techniques over a non-Gaussian indoor WLAN environment, which can be accurately characterized by the JFTS model. We focus on the CDF based approach [9] for deriving ABER expressions. We also study the impact of different JFTS distribution parameters on the performance based on numerical results.

- In this paper, we provide expressions that are numerically efficient and simple to handle. Towards this end, we derive error rate expressions that involve truncation of the infinite series of the original expressions. The approximate expressions are derived in a way such that they guarantee that the area under the probability density function (PDF) of the instantaneous SNR remains equal to 1 . However, the truncation bound is decided based on the study done in [10] for analysis of bandwidth efficiency for the JFTS channel.

- We also evaluate the channel cutoff rate associated with $M$-ary signaling and JFTS channels both in presence and absence of channel magnitude and phase estimates. The channel cutoff rate $R_{0}$ of the communication link is defined as a channel capacity related quantity such that for any $R_{c}<R_{0}$, it is possible to construct a channel code using block length $n$ and coding rate $R_{c}$ capable of maintaining an average error probability less than or equal to $2^{-n\left(R_{0}-R_{c}\right)}$ [11]. Quantifying the channel cutoff rate is done using the Chernoff bound calculation. Therefore, the channel cutoff rate obtained in this way can also be used as an analytical bound limiting the bit error perfor $M$-ary signaling techniques are implemented.

The paper is organized as follows. Section II describes the statistics of instantaneous received SNR over a JFTS faded/shadowed channel. The error performance and channel capacity analyses are presented in Section III and Section IV. Numerical results and discussions are given in Section $\mathrm{V}$, while concluding remarks are provided in Section VI.

\section{Statistics OF Instantaneous SNR}

Let $s(k)$ represent the transmitted data symbol with symbol energy $E_{s}=\mathrm{E}\left\{|s(k)|^{2}\right\}$ that is transmitted over a composite slow shadowed and flat faded wireless communication channel with JFTS statistics. The bit energy is then defined as $E_{b}=$ $E_{S} / \log _{2} M$, where $M$ is the modulation constellation size used for data transmission. The received data symbol $y(k)$ over the sampling instant $k$ can be given by

$$
y(k)=z(k) e^{J \phi(k)} s(k)+n(k)
$$

where $n(k)$ is the complex Additive White Gaussian Noise (AWGN) with one side power spectral density of $N_{0}$, $\phi(k)$ is the instantaneous phase and $z(k)$ denotes the composite fading/shadowing envelope which is JFTS distributed.

The first order statistics of the channel joint fading/ shadowing stochastic process, $z(k)$, can be represented by the

random variable, $Z$, which has a PDF given by [4]

$$
\begin{aligned}
f_{Z}(z)= & \sum_{i=1}^{4} \sum_{h=1}^{m} \frac{b_{i} z R_{h}}{2 P_{1} P_{2}} e^{-K-S_{h}-\frac{z^{2}}{2 P_{2} r_{h}^{2}}} \\
\times & {\left[C_{1 i} I_{0}\left(2 z \sqrt{\frac{K S_{h}\left(1-\Delta T_{i}\right)}{P_{1} P_{2}}}\right)\right.} \\
& \left.+C_{2 i} I_{0}\left(2 z \sqrt{\frac{K S_{h}\left(1+\Delta T_{i}\right)}{P_{1} P_{2}}}\right)\right]
\end{aligned}
$$

and a CDF given by [7]

$$
\begin{aligned}
F_{Z}(z)= & \sum_{i=1}^{4} \sum_{h=1}^{m} \frac{b_{i} R_{h} r_{h} e^{-K-S_{h}}}{2 P_{1} \sqrt{P_{2}}} \\
& \times\left[C_{1 i} Q_{l}\left(\sqrt{\frac{K S_{h}\left(1-\Delta T_{i}\right) r_{h}}{P_{1}}}, z\right)\right. \\
& \left.+C_{2 i} Q_{l}\left(\sqrt{\frac{K S_{h}\left(1+\Delta T_{i}\right) r_{h}}{P_{1}}}, z\right)\right]
\end{aligned}
$$

where $C_{1 i}=e^{S_{h} \Delta T_{i}}, C_{2 i}=e^{-S_{h} \Delta T_{i}}, T_{i}=\cos ((i-1) \pi / 7)$, $I_{0}$ is the 0th-order modified Bessel function of the first kind, $m$ is the quadrature order, $R_{h}=\frac{w_{h}}{\left|r_{h}\right|} e^{r_{h}^{2}-r_{h}^{2} / 2 P_{1}}, w_{h}$ are the Gauss-Hermite quadrature weight factors [12], $r_{h}$ are the roots of the Hermite polynomial for $h=1,2, \ldots, m, Q_{1}$ is the Marcum $Q$-function and $b_{i}=a_{i} I_{0}(1)$ with $a_{1}=\frac{751}{17280}$, $a_{2}=\frac{3577}{17280}, a_{3}=\frac{49}{640}$ and $a_{4}=\frac{2989}{17280}$. The behavior of the JFTS distribution is guided by the fading parameter $K$, shadowing parameter $S_{h}$, shape parameter $\Delta$ and mean-squares of the diffused and the shadowed components, $P_{1}$ and $P_{2}$, respectively. For our analysis we have chosen the quadrature order $m=20$.

If the instantaneous received SNR per symbol is defined as $\gamma(k)=z^{2}(k) E_{s} / N_{0}$ and the average received SNR as $\bar{\gamma}(k)=$ $\mathrm{E}\left\{z^{2}(k)\right\} E_{s} / N_{0}$, where $E_{s}$ is the energy per symbol and $N_{0}$ is the noise density (the noise power present in $1 \mathrm{~Hz}$ ), the PDF of $\gamma$ (for the sake of simplicity of notation, we drop the sampling index $k$ from here on) can be obtained in terms of $\bar{\gamma}[13]$ by change of variables as

$$
\begin{aligned}
f_{\gamma}(\gamma)= & \sum_{i=1}^{4} \sum_{h=1}^{m} \frac{b_{i} R_{h} \Omega}{\bar{\gamma} P_{1} P_{2}} e^{-K-S_{h}-\frac{\Omega \gamma}{2 \bar{\gamma} P_{2} r_{h}^{2}}} \\
& \times\left[C_{1 i} I_{0}\left(2 \sqrt{\frac{K S_{h}\left(1-\Delta T_{i}\right) \gamma \Omega}{\bar{\gamma} P_{1} P_{2}}}\right)\right. \\
& \left.+C_{2 i} I_{0}\left(2 \sqrt{\frac{K S_{h}\left(1+\Delta T_{i}\right) \gamma \Omega}{\bar{\gamma} P_{1} P_{2}}}\right)\right]
\end{aligned}
$$

146 147 148

where $\Omega$ is the mean-squared value of the JFTS envelope given by $\Omega=4 P_{1} P_{2}(1+K)\left(1+S_{h}\right)$ [6]. The corresponding CDF of $\gamma$ can be defined as $F_{\gamma}(\gamma) \triangleq \int_{-\infty}^{\gamma} f_{\gamma}(u) \mathrm{d} u$ and can be expressed as $F_{\gamma}(\gamma)=F_{z}\left(\sqrt{\gamma \mathrm{E}\left\{Z^{2}\right\} / \bar{\gamma}}\right)$.

It is to be noted that owing to the complexity of (4), further integration over $f_{\gamma}(\gamma)$ may not produce a mathematically tractable expression. Hence, we may need an approximate alternative expression for $f_{\gamma}(\gamma)$. Given the infinite series 
expansion of modified Bessel function, $I_{0}(f)=\sum_{t=0}^{\infty} \frac{(f / 2)^{2 t}}{(t !)^{2}}$, we arrive at an alternative expression for (4) as

$f_{\gamma}(\gamma)=\sum_{i=1}^{4} \sum_{h=1}^{m} \sum_{t=0}^{\infty} \frac{A_{i, h}}{\bar{\gamma}(t !)^{2}} e^{-B_{h} \gamma / \bar{\gamma}}$

$$
\times\left[C_{1 i}\left(C_{3 i} \gamma / \bar{\gamma}\right)^{t}+C_{2 i}\left(C_{4 i} \gamma / \bar{\gamma}\right)^{t}\right]
$$

where $A_{i, h}=\frac{b_{i} R_{h} \Omega}{P_{1} P_{2}} e^{-K-S_{h}}, B_{h}=\frac{\Omega}{2 P_{2} r^{2}}, C_{3 i}=K S_{h}(1-$ $\left.\Delta T_{i}\right) \Omega /\left(P_{1} P_{2}\right)$ and $C_{4 i}=K S_{h}\left(1+\Delta T_{i}\right) \Omega /\left(P_{1} P_{2}\right)$. If we truncate the infinite series summation to $t_{\max }$, we can obtain an approximate version of (5) as below,

$$
\begin{aligned}
f_{\gamma}(\gamma) \approx \sum_{i=1}^{4} \sum_{h=1}^{m} & \sum_{t=0}^{t_{\max }} \frac{A_{i, h}}{\bar{\gamma}(t !)^{2}} e^{-B_{h} \gamma / \bar{\gamma}} \\
& \times\left[D_{1 t} C_{1 i}\left(C_{3 i} \gamma / \bar{\gamma}\right)^{t}+D_{2 t} C_{2 i}\left(C_{4 i} \gamma / \bar{\gamma}\right)^{t}\right]
\end{aligned}
$$

where $D_{1 t}=\frac{\bar{\gamma}(t !)^{2}}{A_{i, h}}\left(\frac{\bar{\gamma}}{C_{3 i}}\right)^{t} \sum_{u=1}^{t_{\max }+1}(u-1) !\left(\frac{\bar{\gamma}}{B_{h}}\right)^{u}$ and $D_{2 t}=$ $\frac{\bar{\gamma}(t !)^{2}}{A_{i, h}}\left(\frac{\bar{\gamma}}{C_{4 i}}\right)^{t} \sum_{u=1}^{t_{\max }+1}(u-1) !\left(\frac{\bar{\gamma}}{B_{h}}\right)^{u}$ for $\gamma>0$ and $B_{h}>0$. The constants, $D_{1 t}$ and $D_{2 t}$ in (6) are derived for the sake of guaranteeing that the area under the PDF of $\gamma$ remains equal to 1 . For our numerical analysis, we have chosen $t_{\max }=$ 25 based on the observations in [10].

Proposition 1: The exact and approximate expressions for $F_{\gamma}(\gamma)$ can be given by

$$
\begin{aligned}
F_{\gamma}(\gamma)=1-\left[\sum_{i=1}^{4} \sum_{h=1}^{m} \sum_{t=0}^{\infty} \frac{A_{i, h} \Gamma\left(t+1, B_{h} \gamma / \bar{\gamma}\right)}{(t !)^{2} B_{h}^{t+1}}\right. \\
\left.\times\left(C_{1 i} C_{3 i}^{t}+C_{2 i} C_{4 i}^{t}\right)\right] \\
\approx 1-\left[\sum_{i=1}^{4} \sum_{h=1}^{m} \sum_{t=0}^{t_{\max }} \frac{A_{i, h} \Gamma\left(t+1, B_{h} \gamma / \bar{\gamma}\right)}{(t !)^{2} B_{h}^{t+1}}\right. \\
\left.\times\left(D_{1 t} C_{1 i} C_{3 i}^{t}+D_{2 t} C_{2 i} C_{4 i}^{t}\right)\right]
\end{aligned}
$$

where $\Gamma(\cdot, \cdot)$ is the generalized upper incomplete Gamma function [12].

Proof: See Appendix A.

In the next section, we will be using the CDF-based approach and therefore, (7) for deriving the ABER expressions for different modulation techniques over a JFTS faded/shadowed communication channel.

\section{ERror PERformance ANALysis}

In order to obtain the ABER of a large variety of modulation techniques, the CDF-based approach of [9] and [14] will be used in this section.

\section{A. Binary Modulation Schemes}

For any binary coherent and noncoherent modulation technique, the ABER over a composite flat faded and slow shadowed wireless communication channel suffering from AWGN can be expressed in terms of the CDF of the instantaneous SNR as [9]

$$
P_{b}^{\text {Binary }}(e)=\frac{\alpha^{\beta}}{2 \Gamma(\beta)} \int_{0}^{\infty} \gamma^{\beta-1} e^{-\alpha \gamma} F_{\gamma}(\gamma) \mathrm{d} \gamma
$$

where $\alpha=1$ for Binary Phase Shift Keying (BPSK) and ${ }_{216}$ $\alpha=1 / 2$ for Binary Frequency Shift Keying (BFSK). If the modulation is differential or noncoherent, $\beta=1$, while for coherent modulation, $\beta=1 / 2$.

Proposition 2: The ABER expression for any coherent or noncoherent binary modulation technique over a JFTS faded/shadowed channel can be obtained in exact and approximate mathematically tractable format as

$$
\begin{aligned}
& P_{b}^{\text {Binary }}(e) \\
&=\frac{1}{2}-\left[\sum_{i=1}^{4} \sum_{h=1}^{m} \sum_{t=0}^{\infty} \frac{A_{i, h}}{(t !)^{2}}\left(C_{1 i} C_{3 i}^{t}+C_{2 i} C_{4 i}^{t}\right)\right. \\
& \quad \times \frac{\alpha^{\beta} \bar{\gamma}^{\beta}}{2 \beta \Gamma(\beta)} \frac{\Gamma(\beta+t+1)}{\left(\alpha \bar{\gamma}+B_{h}\right)^{\beta+t+1}} \\
&\left.\quad \times{ }_{2} F_{1}\left(1, \beta+t+1 ; \beta+1 ; \frac{\alpha \bar{\gamma}}{\alpha \bar{\gamma}+B_{h}}\right)\right] \\
& \approx \frac{1}{2}-\left[\sum_{i=1}^{4} \sum_{h=1}^{m} \sum_{t=0}^{t_{\max }} \frac{A_{i, h}}{(t !)^{2}}\left(D_{1 t} C_{1 i} C_{3 i}^{t}+D_{2 t} C_{2 i} C_{4 i}^{t}\right)\right. \\
& \times \frac{\alpha^{\beta} \bar{\gamma}^{\beta}}{2 \beta \Gamma(\beta)} \frac{\Gamma(\beta+t+1)}{\left(\alpha \bar{\gamma}+B_{h}\right)^{\beta+t+1}} \\
&\left.\times{ }_{2} F_{1}\left(1, \beta+t+1 ; \beta+1 ; \frac{\alpha \bar{\gamma}}{\alpha \bar{\gamma}+B_{h}}\right)\right] . \quad{ }^{228}
\end{aligned}
$$

where ${ }_{p} F_{q}(\cdot)$ is the generalized hyper-geometric function [12] and $p, q$ are integers.

Proof: See Appendix B.

\section{B. M-Ary Coherent Modulation Schemes}

In order to evaluate the error performance of $M$-ary coherent modulation techniques over a composite fading/shadowing channel, we need to calculate an integral of the form [9]

$$
P_{b}^{M \text {-ary }}(e, g)=\frac{1}{\sqrt{2 \pi}} \int_{0}^{\infty} F_{\gamma}\left(\frac{v^{2}}{g}\right) e^{-v^{2} / 2} \mathrm{~d} v
$$

where $g$ depends on the modulation type [13].

Proposition 3: For a JFTS channel, ABER expression for coherent $M$-ary modulation technique can be obtained in exact and approximate mathematically tractable format as

$$
\begin{aligned}
P_{b}^{M \text {-ary }}(e, g) & \frac{1}{2}-\left[\sum_{i=1}^{4} \sum_{h=1}^{m} \sum_{t=0}^{\infty} \frac{A_{i, h}}{(t !)^{2}}\left(C_{1 i} C_{3 i}^{t}+C_{2 i} C_{4 i}^{t}\right)\right. \\
& \left.\times \frac{2^{t+1} \sqrt{g \bar{\gamma}} \Gamma(t+3 / 2)}{\left(g \bar{\gamma}+2 B_{h}\right)^{t+3 / 2}}{ }_{2} F_{1}\left(1, t+\frac{3}{2} ; \frac{3}{2} ; \frac{g \bar{\gamma}}{g \bar{\gamma}+2 B_{h}}\right)\right] \\
\approx & \frac{1}{2}-\left[\sum_{i=1}^{4} \sum_{h=1}^{m} \sum_{t=0}^{t_{\max }}\left(D_{1 t} C_{1 i} C_{3 i}^{t}+D_{2 t} C_{2 i} C_{4 i}^{t}\right) \frac{A_{i, h}}{(t !)^{2}}\right. \\
& \left.\times \frac{2^{t+1} \sqrt{g \bar{\gamma}} \Gamma(t+3 / 2)}{\left(g \bar{\gamma}+2 B_{h}\right)^{t+3 / 2}}{ }_{2} F_{1}\left(1, t+\frac{3}{2} ; \frac{3}{2} ; \frac{g \bar{\gamma}}{g \bar{\gamma}+2 B_{h}}\right)\right] .
\end{aligned}
$$

Proof: See Appendix C.

It is evident that bit error rate is heavily driven by the factors $C_{3 i}$ and $C_{4 i}$. Both terms are directly proportional to 
the product of the fading parameter $K$ and the shadowing parameter $S_{h}$. Hence, when $K, S_{h}$, or both increases, the ABER performance improves. It can also be concluded that ABER is also influenced by the $\Delta$-parameter. However, exactly how much ABER is affected by a change in $\Delta$ is difficult to predict strictly by inspecting the ABER expressions. The sensitivity to changes in $\Delta$ is best evaluated using the ABER plots presented in Section V.

1) M-Ary Quadrature Amplitude Modulation (M-QAM): Using unified approximation, as is done in [13], the ABER expression of general order $M$-QAM modulation over a composite fading / shadowing channel is given by [9]

$$
P_{b}^{M-\mathrm{QAM}}(e) \cong \frac{4}{\log _{2} M}\left(1-\frac{1}{\sqrt{M}}\right) \sum_{n=1}^{\sqrt{M} / 2} P_{b}^{M \text {-ary }}\left(e, g_{n-Q}\right)
$$

where $g_{n-Q}=3(2 n-1)^{2} \log _{2} M /(M-1)$. The ABER expression for general order $M$-QAM over JFTS channel can be expressed by substituting (11) in (12) and putting $g=g_{n-Q}$ in (11).

2) M-Ary Phase Shift Keying (M-PSK): Using unified approximation, as is done in [13], the ABER expression of Gray-coded coherent $M$-PSK modulation over a composite fading / shadowing channel is given by [9]

$$
P_{b}^{M-\mathrm{PSK}}(e) \cong \frac{2}{\max \left(\log _{2} M, 2\right)} \sum_{n=1}^{\max (M / 4,1)} P_{b}^{M \text {-ary }}\left(e, g_{n-P}\right)
$$

where $g_{n-P}=2\left(\log _{2} M\right) \sin ^{2}((2 n-1) \pi / M)$. The ABER expression for coherent $M$-PSK over JFTS channel can be expressed by substituting (11) in (13) and putting $g=g_{n-P}$ in (11).

It is note-worthy that in this paper, we have shown how the JFTS distribution provides mathematically tractable error probability expressions in terms of generalized hypergeometric functions. Such expressions can be obtained only numerically for the commonly used Suzuki / Nakagami - lognormal distributions using log-normal approximations [15]. However, this approximation is only valid for large lognormal spreading factor, $\sigma$. Easy-to-use expressions for ABER of basic modulation techniques exist in literature only for $\mathcal{K}$-fading channels [3] among commonly used fading/ shadowing channel models. Yet even for Gray-coded $M$-PSK, symbol error probability over $\mathcal{K}$-fading channel involves an unsolvable integral which can only be evaluated numerically.

\section{Achievable Capacity Analysis}

It has been argued in [16] that the average capacity measure is only an upper bound on the channel capacity achievable without transmitter feedbacks. Since for a JFTS channel, the average capacity derived in [8] can only be expressed in terms of infinite series summation, it is more appealing to obtain the channel cutoff rate achievable using only variable rate $M$-ary signaling. The channel cutoff rate can be calculated using [11]

$$
R_{0}=2 \log _{2}(M)-\log _{2}\left(\sum_{j=1}^{M} \sum_{l=1}^{M} \mathbb{C}\left(\hat{s}_{j}, s_{j}\right)\right)
$$

where $\mathbb{C}\left(\hat{s}_{j}, s_{j}\right)$ is the Chernoff bound on the pairwise error probability [13] associated with choosing the sequence $\hat{\mathbf{s}}=$ $(\hat{s}[1], \ldots, \hat{s}[L])$ at the receiver when the sequence $\mathbf{s}=$ $(s[1], \ldots, s[L])$ is transmitted over the communication link. The Chernoff bound can be calculated in presence of perfect channel state information (CSI) as [18], [19]

$$
\mathbb{C}_{\mathrm{CSI}}\left(\hat{s}_{j}, s_{j}\right)=\mathrm{E}_{Z}\left\langle e^{-z^{2} \frac{\left|d_{j}\right|^{2}}{4}}\right\rangle \quad \text { [bits/symbol] }
$$

and in absence of any CSI as [18], [19]

$$
\mathbb{C}_{\mathrm{NCSI}}\left(\hat{s}_{j}, s_{j}, \lambda\right)=e^{-\lambda^{2}\left|d_{j}\right|^{2}} \mathrm{E}_{Z}\left(e^{-\lambda z\left|d_{j}\right|^{2}}\right\rangle[\mathrm{bits} / \mathrm{symbol}]
$$

where $\left|d_{j}\right|^{2}=\left|\hat{s}_{j}-s_{j}\right|^{2} / N_{0}, N_{0}$ is the noise spectral density of the corrupting AWGN and $\lambda$ is a nonnegative real valued parameter over which (16) can achieve the tightest possible exponential bound on the achievable cutoff rate in absence of any CSI.

Proposition 4: For a JFTS channel, the Chernoff bound in presence of perfect CSI is given by by putting (5) in (15) and using integral solution from [17, p. 709, eq. (6.643.2)], ${ }^{1}$

$$
\begin{aligned}
\mathbb{C}_{\mathrm{CSI}}\left(\hat{s}_{j}, s_{j}\right) & \\
= & \sum_{i=1}^{4} \sum_{h=1}^{m} \frac{b_{i} R_{h} r_{h}^{2} e^{-K-S_{h}}}{P_{1}\left(2+\left|d_{j}\right|^{2} P_{2} r_{h}^{2}\right)} \\
& \times\left[e^{\frac{8 K S_{h}\left(1-\Delta T_{j}\right) r_{h}^{2}}{P_{1}\left(4+\left|d_{j}\right|^{2} P_{2} r_{h}^{2}\right)}} e^{S_{h} \Delta T_{i}}+e^{-S_{h} \Delta T_{i}+\frac{8 K S_{h}\left(1+\Delta T_{i}\right) r_{h}^{2}}{P_{1}\left(4+\left|d_{j}\right|^{2} P_{2} r_{h}^{2}\right)}}\right]
\end{aligned}
$$

and in absence of any CSI is given by

$$
\begin{aligned}
\mathbb{C}_{\mathrm{NCSI}}\left(\hat{s}_{j}, s_{j}\right) \\
=\sum_{i=1}^{4} \sum_{h=1}^{m} \frac{b_{i} R_{h} e^{-K-S_{h}}}{2 P_{1} P_{2}} \\
\quad \times\left[\int_{0}^{\pi}\left(1-\sqrt{\pi} v_{1} \operatorname{erfc}\left(v_{1}\right) e^{\nu_{1}^{2}+S_{h} \Delta T_{i}}\right) \mathrm{d} \theta\right. \\
\left.\quad+\int_{0}^{\pi}\left(1-\sqrt{\pi} \nu_{2} \operatorname{erfc}\left(\nu_{2}\right) e^{\nu_{2}^{2}-S_{h} \Delta T_{i}}\right) \mathrm{d} \theta\right]
\end{aligned}
$$

where $v_{1}=\lambda d_{j}^{2} \sqrt{\frac{P_{2} r_{h}^{2}}{2\left(1+2 P_{2} r_{h}^{2}\right)}}-\sqrt{\frac{K S_{h}\left(1-\Delta T_{i}\right)}{P_{1} P_{2}}} \cos (\theta), v_{2}=$ $\lambda d_{j}^{2} \sqrt{\frac{P_{2} r_{h}^{2}}{2\left(1+2 P_{2} r_{h}^{2}\right)}}-\sqrt{\frac{K S_{h}\left(1+\Delta T_{i}\right)}{P_{1} P_{2}}} \cos (\theta)$ and $\operatorname{erfc}(u)=$ $\frac{2}{\sqrt{\pi}} \int_{u}^{\infty} e^{-\gamma^{2}} \mathrm{~d} \gamma$ is the complimentary error function.

In absence of CSI, minimizing $\lambda$ in (16) will maximize the cutoff rate. Without side information, no uniform minimization of $\lambda$ exists [19]. In that case, (18) has to be evaluated numerically using the Laguerre-Gauss Quadrature method and substituted back in (17) to derive the expression for the Chernoff factors of JFTS fading/shadowing link without any CSI available at the receiver.

\footnotetext{
${ }^{1}$ As is evident from (17), the channel cutoff rate achievable using $M$-ary signaling over a JFTS faded/shadowed channel will increase with the decrease in the Chernoff bound, which in turn decreases exponentially with the increase in the fading $(K)$ and/or the shadowing $\left(S_{h}\right)$ parameters.
} 
TABLE I

Generalized RANGe of VALUES FOR the PARAMETERS OF JFTS DISTRIBUTION

\begin{tabular}{|l|l|l|l|}
\hline $\begin{array}{l}\text { User / Access } \\
\text { Point (AP) } \\
\text { Positions }\end{array}$ & $K$ (dB) & $S_{h}$ (dB) & $\Delta$ \\
\hline \hline Same room & 9 to 11 & 9 to 12 & 0.6 to 0.95 \\
\hline $\begin{array}{l}\text { Rooms } \\
\text { separated by } \\
1 \text { wall or } \\
\text { partition }\end{array}$ & 7 to 9 & 6 to 11 & 0.3 to 0.58 \\
\hline $\begin{array}{l}\text { Rooms sepa- } \\
\text { rated by 2-3 } \\
\text { walls or parti- } \\
\text { tions }\end{array}$ & 6 to 7 & -4.5 to -0.5 & 0.2 to 0.3 \\
\hline $\begin{array}{l}\text { Rooms sepa- } \\
\text { rated by more } \\
\text { than 3 walls } \\
\text { or partitions }\end{array}$ & 5 to 6 & -5 to -9.8 & 0.1 to 0.2 \\
\hline
\end{tabular}

\section{Numerical Results AND Discussion}

The derived mathematically tractable expressions for the ABER of coherent / noncoherent binary modulation techniques like BPSK, BFSK, general order $M$-QAM and coherently detected $M$-PSK are numerically evaluated and plotted as functions of the parameters of the communication channel model and the modulation techniques. The analytical results are compared with those obtained through Monte Carlo simulation in order to validate the proposed analysis. For plotting the analytical results, we have chosen the approximation index, $m=20$.

The wireless communication channel between the transmitter and the receiver is assumed to be suffering from AWGN and the composite fading / shadowing envelope is assumed to be JFTS distributed, where all the channel samples are statistically independent. The channel samples are generated using JFTS distributed random variables $Z=X Y$ where $X$ are Ricean distributed and $Y$ are TWDP distributed. All the analytical and simulation results are evaluated for single channel receivers and are averaged over 100 independent random channel realizations. The ABER results are plotted as functions of the average SNR per bit $\left(E_{b} / N_{0}\right)$ in decibels.

The behavior of the JFTS distribution is guided by its fundamental parameters $K, S_{h}$ and $\Delta$. The ranges of values for those model parameters that are suitable for different scenarios within the large office indoor wireless environments are compiled in [4]. The particular set of JFTS parameters that are used for numerical analysis and generating simulation results in this paper are enlisted in Table I.

\section{A. Error Probability Performance}

1) Performance of Modulation Techniques: The first set of curves in Fig. 1 are generated for evaluating performance of different modulation techniques over a JFTS fading / shadowing communication channel. Both analytical and simulation

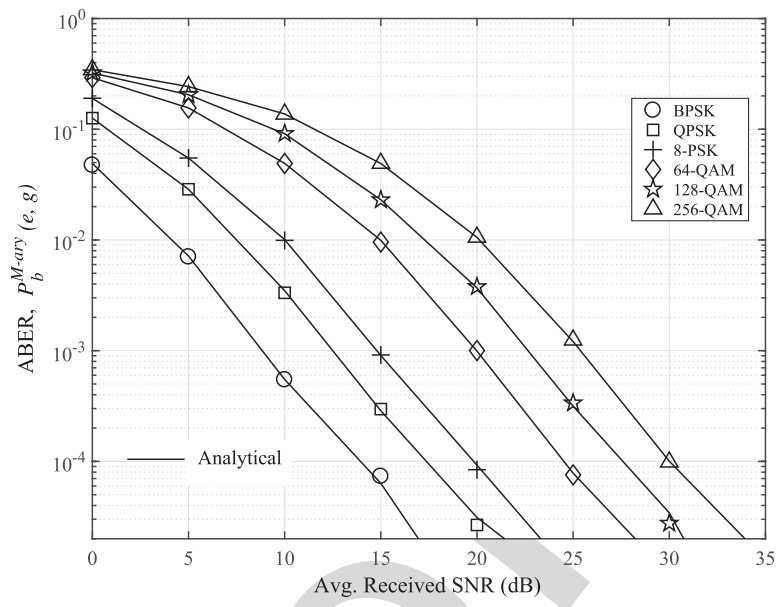

Fig. 1. Comparative simulation and analytical average bit error rate performances of coherent $M$-PSK and $M$-QAM over a JFTS faded / shadowed communication link.

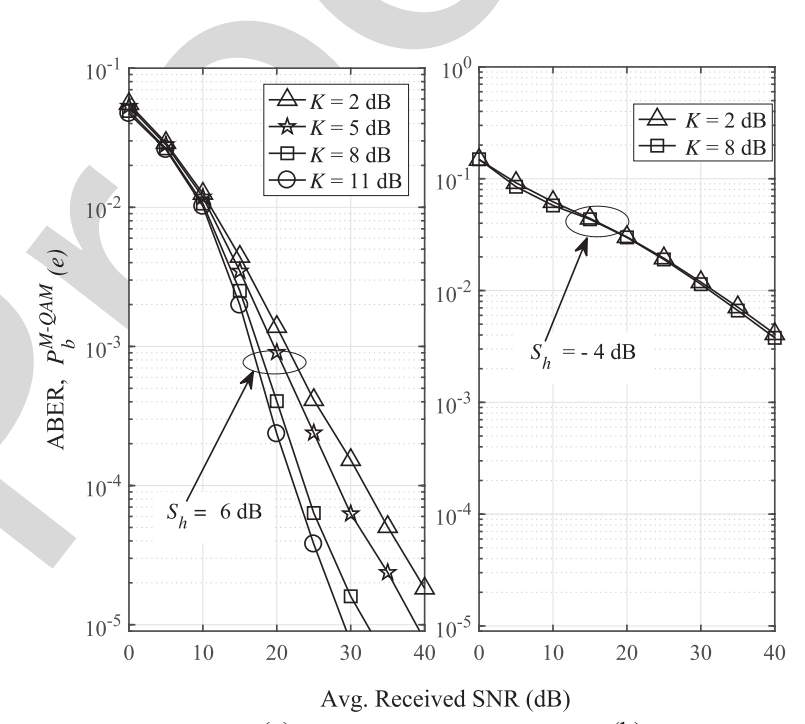

(a)

(b)

Fig. 2. Average simulated bit error rate performances of 16-QAM over JFTS faded / shadowed communication link with (a) $S_{h}=6 \mathrm{~dB}, \Delta=0.3$ and (b) $S_{h}=-4 \mathrm{~dB}, \Delta=0.9$.

results are plotted for each modulation scheme. All the results are generated for a fixed set of JFTS parameters, $K=6.5 \mathrm{~dB}$, $S_{h}=5 \mathrm{~dB}$ and $\Delta=0.8$. This set of parameters are encountered when the mobile WLAN user and the access point are separated by 1 set of dry-wall or partition, as tabulated in Table I. It is evident from Fig. 1 that the derived analytical results from (9) and (11) offer a good agreement with that of the simulation results and they fall within $1-2 \mathrm{~dB}$ of the simulation results. It is to be noted that in many cases analytical results do not exactly match the simulation results as the analytical expressions involve the approximation index $m$.

2) Effect of the JFTS Parameters: The curves in the first subplot of Fig. 2 are generated by varying the $K$-parameter of the JFTS distribution. The other parameters of the JFTS distribution like $S_{h}$ and $\Delta$ are kept constant at $6 \mathrm{~dB}$ and 0.3 respectively, representing a condition where the user and the access point are separated by 1 dry-wall or partition. 


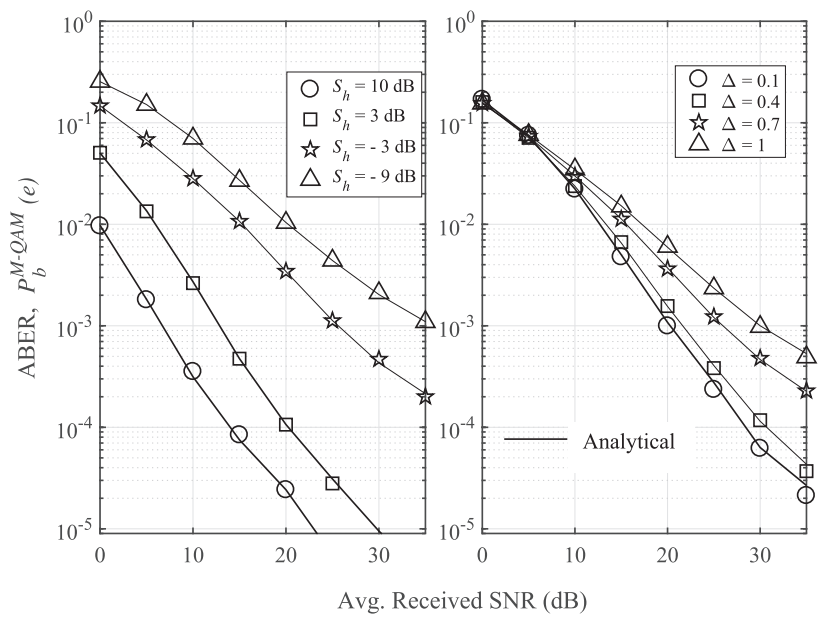

(a)

(b)

Fig. 3. Average bit error rate performances of 16-QAM over JFTS faded / shadowed communication link with (a) $K=6.5 \mathrm{~dB}, \Delta=0.8$ and (b) $K=6 \mathrm{~dB}, S_{h}=-4 \mathrm{~dB}$.

Simulation and analytical ABER performances are plotted for 16-QAM. The performance of 16-QAM deteriorates as the value of $K$ decreases with fixed $S_{h}$ and $\Delta$. This is due to the fact that as $K$ decreases, the power contributed by the strong specular components decreases in comparison to that contributed by the diffused and scattered components, resulting in degradation of overall system performance.

The improvement in performance due to the increase in $K$-factor from $2 \mathrm{~dB}$ to $5 \mathrm{~dB}$ or $5 \mathrm{~dB}$ to $8 \mathrm{~dB}$ is not proportional to the improvement for increasing $K$ from $8 \mathrm{~dB}$ to $11 \mathrm{~dB}$. This is due to the fact that for a JFTS channel, the $S_{h}$ and $\Delta$ parameters also influence the channel behavior. A high $S_{h}$ factor corresponds to a low severity in shadowing. A low $\Delta$ represents a scenario where only one scattering cluster dominates instead of two thereby also resulting in low shadowing severity. Hence, with a high $S_{h}$ and low $\Delta$ coupled with high $K$-factors, the communication channel approaches the "no fading" scenario. As a result changing the $K$-factor from $8 \mathrm{~dB}$ to $11 \mathrm{~dB}$ does not cause any further improvement in performance.

To emphasize the effect of $S_{h}$ and $\Delta$, the ABER performance of 16-QAM is plotted for only $K=2 \mathrm{~dB}$ and $8 \mathrm{~dB}$ in the second subplot of Fig. 2. In this case a low $S_{h}$ factor of $-4 \mathrm{~dB}$ and a high $\Delta$ factor of 0.9 is used. This represents a condition where the user and the access point are separated by $2-3$ partitions. The difference in performance due to the increase in $K$-factor is completely obliterated for this set of shadowing parameters. A low $S_{h}$ and a high $\Delta$ corresponds to a very high severity in shadowing and further degrading fading by a reduction in $K$ does not further degrade performance in any significant way.

Fig. 3(a), is generated by varying the $S_{h}$ parameter of the JFTS distribution with fixed $K$ and $\Delta$ at $6.5 \mathrm{~dB}$ and 0.8 respectively. This set of $K$ and $\Delta$ parameters represent a scenario where the user and the access point are separated by $2-3$ dry-walls or partitions. It is evident from Fig. 3(a) that lowering the $S_{h}$-parameter value results in deteriorated system performance. A larger $S_{h}$ factor represents large variations

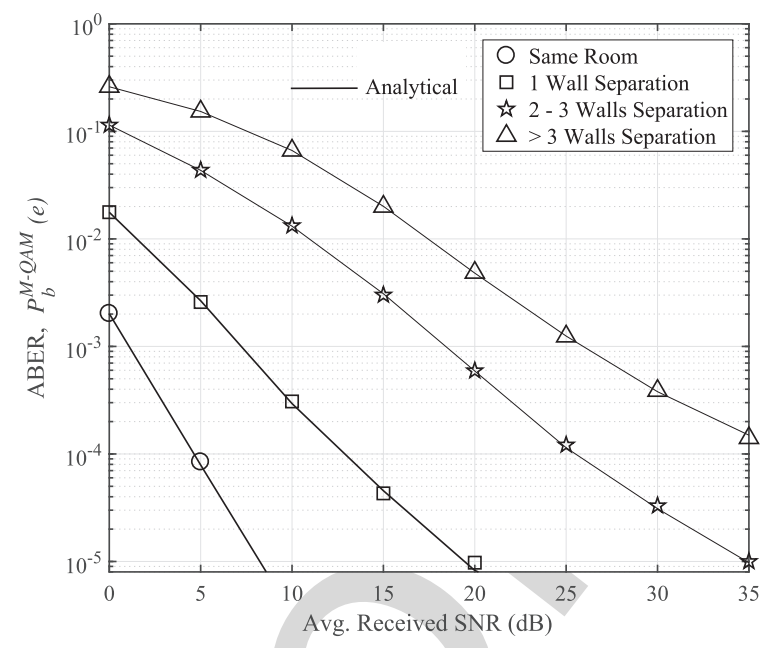

Fig. 4. Average bit error rate performances of 16-QAM over JFTS faded / shadowed communication link, where the curves are generated by varying all the JFTS parameters, $K, S_{h}$ and $\Delta$ simultaneously.

in the main wave amplitudes contributed by each scattering neighborhood resulting in approximately equable number of high and low, thereby reducing the overall severity of shadowing. On the other hand, a low $S_{h}$ factor depicts a scenario where each scattering cluster contributes a very small range of discrete shadowing values, higher in magnitude and encountered repeatedly. This condition results in an increased severity in shadowing, thereby degrading overall system performance.

The curves in Fig. 3(b) are generated by varying only the $\Delta$-parameter of the JFTS distribution but keeping a fixed $K$ and $S_{h}$ at $6 \mathrm{~dB}$ and $-4 \mathrm{~dB}$ respectively. This set of $K$ and $S_{h}$ is encountered when the mobile WLAN user and the access point are separated by $2-3$ dry-walls or partitions. Variations in the value of the $\Delta$-parameter affects the system performance in a way opposite to that of the $K$ or $S_{h}$ parameter. Performance of 16-QAM degrades as $\Delta$ increases from 0.1 to 0.7 . This is due to the fact that as $\Delta$ increases, the relative magnitudes of the shadowing values contributed by two successive scattering clusters visited by the user increases. This results in an increase in the shadowing severity.

The results in Fig. 4 are generated by varying the JFTS parameters, $K, S_{h}$ and $\Delta$. The values for each set of parameters are chosen from the ranges of their numerical values proposed in Table I, depending on the relative position of the mobile LAN user and the access point. The first curve is generated for $K=10 \mathrm{~dB}, S_{h}=10.5 \mathrm{~dB}$ and $\Delta=0.75$, representing a condition where both the user and the access point are located in the same room Table I. As the user moves to a different room separated by one set of wall or partition from that of the access point $\left(K=8 \mathrm{~dB}, S_{h}=6.5 \mathrm{~dB}\right.$ and $\Delta=0.45)$, system performance degrades, as exhibited by the second curve in Fig. 4. In order to achieve an ABER of $10^{-3}$, the average SNR per symbol requirement increases by around $5 \mathrm{~dB}$ for the second set of parameters with respect to the first set of JFTS parameters. It should be emphasized that Fig. 4 assumes a common path loss for all four scenarios. This means that for a given SNR, all four scenarios experience the same average path loss and the difference in BER shown on the plot 


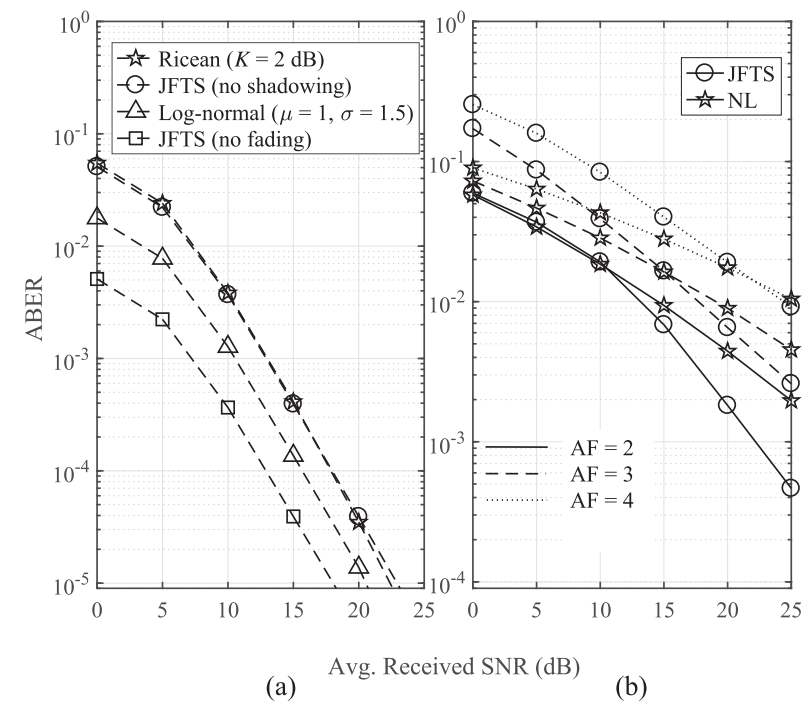

Fig. 5. Comparative average bit error rate of (a) QPSK over JFTS link with that over traditional channel models and (b) 16-QAM over a JFTS link with that over composite Nakagami - log-normal (NL) link.

is a result of the different small-scale fading and shadowing statistics imposed by the JFTS model.

The average SNR per symbol requirement increases even further by around $10 \mathrm{~dB}$, if the user and the access point is separated by $2-3$ sets of partitions $(K=6.5 \mathrm{~dB}$, $S_{h}=-1.5 \mathrm{~dB}$ and $\left.\Delta=0.25\right)$. This happens due to the lack of strong specular components and the presence of at least two scattering clusters between the transmitter and the receiver, which jointly deteriorates the overall system performance. However, for propagation conditions that correspond to an increase in the number of separations by more than 3 (i.e. $K=5.5 \mathrm{~dB}, S_{h}=-7.5 \mathrm{~dB}, \Delta=0.15$ ), the average SNR per symbol requirement only increases by a maximum of $5 \mathrm{~dB}$, in order to achieve the same ABER performance. The reason for this can be imparted to the low $\Delta$-factor, where the effect of one scattering cluster is much stronger than the other one. As a result, system performance is effectively affected by only one scattering cluster, even in the presence of at least two scattering clusters.

3) Comparison With Outdoor Channel Models: To demonstrate how JFTS channel performance compares to conventional channel models, Fig. 5(a) is used to compare performances of QPSK over JFTS, Ricean and log-normal distributed communication channels. We have chosen the Ricean distribution $(K=2 \mathrm{~dB})$ to compare its impact on performance with that of the JFTS channel with no shadowing condition $\left(K=2 \mathrm{~dB}, S_{h}=1000 \mathrm{~dB}, \Delta=0\right)$. The log-normal distribution $(\mu=1, \sigma=1.5)$ is selected to compare with the special case of JFTS channel with no fading $(K=1000 \mathrm{~dB}$, $S_{h}=-3 \mathrm{~dB}, \Delta=0.2$ ). These particular set of JFTS parameters is selected for comparison as they correspond to a TWDP distribution with the same mean and standard deviation. The performance over Ricean channel is almost equivalent to the no shadowing JFTS case, as with $\Delta=0$, the JFTS distribution reduces approximately to Ricean distribution. However, there exists a small difference in performance owing to the minute amount of shadowing still present in the JFTS channel with
$S_{h}=1000 \mathrm{~dB}$. However, that is not the case with the log-normal and the no fading JFTS channels. This is because, putting a very high $K$ in the JFTS distribution reduces it approximately to the TWDP distribution accounting for maximum of two scattering clusters. The TWDP distribution is very different from the log-normal distribution which accounts for the transition through several local neighborhoods, each of which consisting of different clusters of scattering objects.

To illustrate how the JFTS channel performance compares to more conventional outdoor composite fading/shadowing channel models, Fig. 5(b) is used to compare the performance of 16-QAM over JFTS and Nakagami - log-normal (NL) faded / shadowed communication channels. We have chosen the NL distribution, as the Nakagami- $m$ can be varied to model a variety of fading distributions including the Rayleigh and the Ricean distributions. The channel parameters are chosen so that the JFTS and NL channels contribute the same amount of fading (AF). As shown in [6], a JFTS distribution with $K=7 \mathrm{~dB}, S_{h}=6 \mathrm{~dB}$ and $\Delta=0.7$, contributes an $\mathrm{AF}$ of 2 which is same as that contributed by NL distribution with $m=1$ and $\sigma=2.8(4.4 \mathrm{~dB})$. For these sets of parameters, performance over a JFTS channel is worse than that over a NL channel for SNRs less than $10 \mathrm{~dB}(\bar{\gamma} \leq 10 \mathrm{~dB})$. For higher SNRs per bit of around $10 \mathrm{~dB}$ and more, JFTS offers a performance gain over NL. While only the simulation results are shown for brevity, this same pattern in performance repeats itself even for the set of JFTS $\left(K=5,2 \mathrm{~dB}, S_{h}=\right.$ $-5,-10 \mathrm{~dB}, \Delta=0.3,0.5)$ and $\mathrm{NL}(m=1,1, \mu=1,1$, $\sigma=3.6,4.2(5.5,6.2 \mathrm{~dB}))$ parameters that contribute AFs of 3 and 4 respectively. This improvement in performance for the JFTS distribution at higher SNRs occurs due to the fact that for the JFTS distribution, there still exists a very small group of specular components as long as $K \neq 0$. While, $m=1$ for NL distribution represents a small scale fading condition, which is equivalent to Rayleigh fading with the absence of any specular component.

In addition, in order to visualize the difference between JFTS and NL distributions, Fig. 6 exhibits their PDFs. The model parameters for both the distributions are selected such that they contribute the same amount of AF. The PDF of a NL distributed variable $w$ is given by

$f_{W}(w)=\int_{0}^{\infty} \frac{m^{m} w^{m-1}}{v^{m} \Gamma(m)} e^{-\frac{m w}{v}} \frac{4.3429}{\sqrt{2 \pi} \sigma v} e^{-\frac{\left(10 \log _{10} v-\mu\right)^{2}}{2 \sigma^{2}}} \mathrm{~d} v$

where $m$ is the Nakagami $m$-factor, and $\mu$ and $\sigma$ are the mean and standard deviation of the log-normal shadowing distribution, respectively. It is evident from Fig. 6 that the NL distribution fails to adequately characterize indoor large office wireless communication scenarios where a mobile user can traverse through at most two distinct scattering clusters within a time-frame of interest.

\section{B. Achievable Capacity Analysis}

1) Effect of the JFTS Parameters: The effect of the JFTS parameters on channel cutoff rate is shown in Fig. 7. Channel cutoff rate of $M$-ary signaling over a JFTS channel deteriorates with the decrease in $K$ and $S_{h}$-factors. The degradation in cutoff rate due to the decrease in $K$-factor from $8 \mathrm{~dB}$ to $2 \mathrm{~dB}$ 


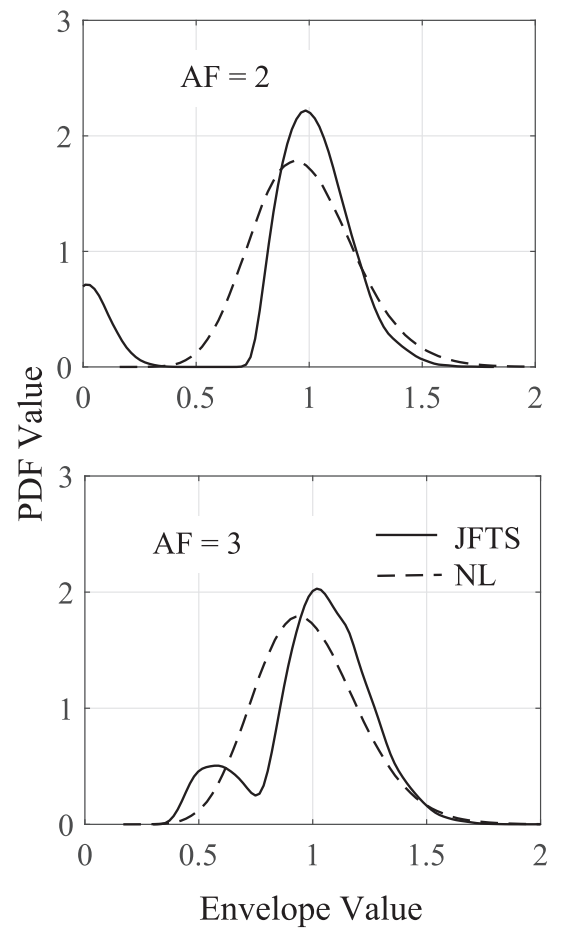

Fig. 6. Comparative PDFs of JFTS and NL distributions (a) AF $=2$ (JFTS : $K=7 \mathrm{~dB}, S_{h}=6 \mathrm{~dB}$ and $\Delta=0.7, \mathrm{NL}: m=1$ and $\left.\sigma=2.8\right)$ and (b) $\mathrm{AF}=3$ (JFTS : $K=5 \mathrm{~dB}, S_{h}=-5 \mathrm{~dB}, \Delta=0.3, \mathrm{NL}: m=1, \mu=1$, $\sigma=3.6$ ).

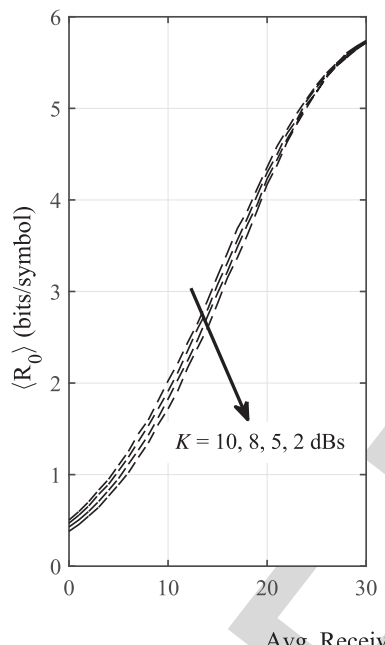

(a)

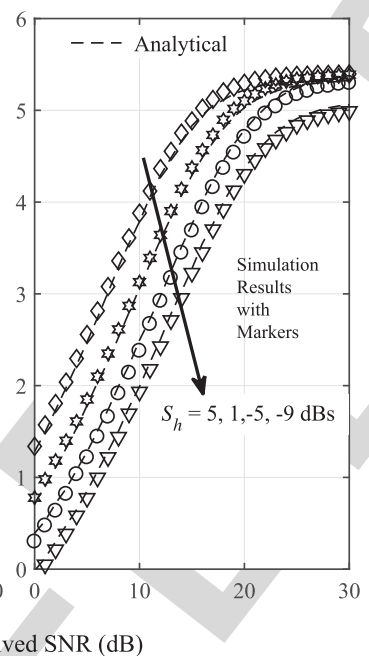

(b)
Fig. 7. Channel cutoff rate, $\left\langle R_{0}\right\rangle$ of JFTS channel (with CSI) as a function of average received signal-to-noise ratio (a) with fixed $S_{h}=1 \mathrm{~dB}, \Delta=0.4$ and (b) with fixed $K=5 \mathrm{~dB}, \Delta=0.9$.

(refer to Fig. 7(a)) is much less compared to the decrease in achievable cutoff rate due to the lowering of $S_{h}$-factor from $5 \mathrm{~dB}$ to $-5 \mathrm{~dB}$ (refer to Fig. 7(b)). These results do not agree with the observations made in [20], where bit error rate performance of BPSK is found to degrade equally either due to the decrease in the $K$-factor or the $S_{h}$-factor. The reason for this can be attributed to the $\Delta$-value chosen for each plot. For Fig. 7(a), a low $\Delta$ of 0.4 is chosen. In this case, shadowing severity is reduced by the fact that only one scattering cluster dominates instead of two clusters. For Fig. 7(b) a high $\Delta$ of 0.9 is chosen, where the magnitudes of the shadowing values contributed by each scattering cluster are almost equal.

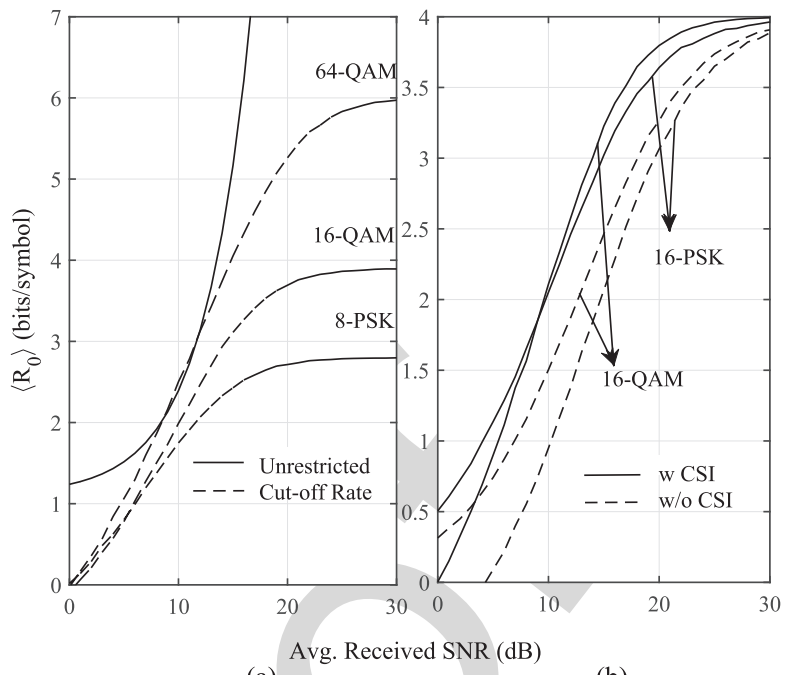

(a)

(b)

Fig. 8. Channel cutoff rate, $\left\langle R_{0}\right\rangle$ of JFTS channel under different modulation techniques (a) with different constellation size $M$ and (b) presence/absence of CSI with $K=7 \mathrm{~dB}, S_{h}=6 \mathrm{~dB}$ and $\Delta=0.7$.

It is worth mentioning that the channel cutoff results plotted in Fig. 7 are analytical results, as these results are only estimates of the achievable throughput, neither exact nor approximate bounds on the actual system performance. However, we have plotted the achievable throughput over JFTS channel with $K=5 \mathrm{~dB}, S_{h}=-9 \mathrm{~dB}, \Delta=0.9$ through Monte-Carlo simulation for comparison. Plotting simulation results for all values of $K$ and $S_{h}$ have been avoided due to space constraint.

2) Performance of Modulation Techniques: Fig. 8 demonstrates the cutoff rate for different modulation schemes in the JFTS channel. The curves in Fig. 8(a) are used to compare performances of $M$-QAM/PSK over JFTS faded / shadowed communication links with $K=7 \mathrm{~dB}, S_{h}=6 \mathrm{~dB}$ and $\Delta=0.7$. The channel cutoff rate curves are generated using (17). The unrestricted upper bound or the average achievable channel capacity under this particular channel condition is also plotted for reference. In this case, the unrestricted upper bound on achievable channel capacity over a JFTS fading/shadowing channel can be evaluated using $C_{\mathrm{JFTS}}=\mathrm{E}_{Z}\left\langle B \tau \log _{2}(1+\right.$ $z \gamma)\rangle$ [bits/symbol], where the expectation is taken over $z$, $B$ is the bandwidth of the input waveform and the waveform is limited to the time interval, $0 \leq t \leq \tau$. The expression for $C_{\mathrm{JFTS}}$ in terms of the JFTS parameters has been derived in $[10$, p. 10075 , eq. (3)].

It is harder to approach the capacity of the JFTS channel compared to the traditional fading channels like Rayleigh channel in the context of QAM, as a consequence of having wider SNR gap between the capacity bound and the cutoff rate. For example, at a capacity of 3 bits/symbol, the SNR gap between the capacity curve and cutoff rate curve of 16-QAM when communicating over the JFTS and the Rayleigh channels is about $12 \mathrm{~dB}$ (refer to Fig. 8(b)) and $5 \mathrm{~dB}$ [21], respectively. For this particular set of curves, we have used a high $K$-factor of $7 \mathrm{~dB}$ as compared to the Rayleigh fading condition $(K=0)$. Hence, in this case, the lower achievable channel capacity over the JFTS channel is a direct impact 
of the presence of shadowing. Therefore, though there still exists a group of waves arriving directly at the receiver over the LOS path, they are obstructed due to the presence of in-building structures like the thin set of dry-walls between the transmitter and the receiver.

However, for the JFTS channel, QAM without CSI still performs better than PSK, as is the case in the presence of perfect CSI (refer to Fig. 8(b)). This contrasting behavior between outdoor and indoor channels can be attributed to that fact that in an indoor environment, shadowing varies quickly enough to have an impact on the decision region boundaries along with fading. In case of PSK receivers, the decision boundaries are not independent of the shadowing depth and therefore performs equally poorly as QAM receivers over a JFTS faded/shadowed communication link.

\section{Conclusions}

The primary contribution of this paper is to derive easy-toevaluate closed-form analytical expressions for the ABER of a wireless communication system using $M$-QAM and coherent $M$-PSK modulation techniques over JFTS fading / shadowing channels. In order to do so, closed-form expressions for the $\mathrm{PDF}$ and the CDF of the received instantaneous composite signal-to-noise ratio (SNR) are utilized. The derived analytical expressions for ABER are numerically evaluated and plotted as functions of the parameters of the communication channel model and the modulation techniques. Performance degrades as $K$ and $S_{h}$ decreases and enhances as $\Delta$ decreases. The analytical results are found to be in agreement with the simulation results verifying the validity of the derived expressions. It can also be concluded that for higher SNRs, performance over a JFTS channel model is better than the NL channel model for the same AF.

\section{APPENDIX A}

\section{PROOF OF PROPOSITION 1}

The CDF of $\gamma$ can be defined as

$$
F_{\gamma}(\gamma) \triangleq \int_{-\infty}^{\gamma} f_{\gamma}(u) \mathrm{d} u=1-\int_{\gamma}^{\infty} f_{\gamma}(u) \mathrm{d} u .
$$

Putting (5) back in (A.20), we can rewrite (A.20) mathematically as

$$
\begin{array}{r}
F_{\gamma}(\gamma)=1-\left[\sum_{i=1}^{4} \sum_{h=1}^{m} \sum_{t=0}^{\infty} \frac{A_{i, h}}{\bar{\gamma}(t !)^{2}}\left(C_{1 i} \frac{C_{3 i}^{t}}{\bar{\gamma}^{t}}+C_{2 i} \frac{C_{4 i}^{t}}{\bar{\gamma}^{t}}\right)\right. \\
\left.\times \int_{\gamma}^{\infty} u^{t} e^{-B_{h} \frac{u}{\gamma}} \mathrm{d} u\right] .
\end{array}
$$

Using the integral solution from [17, p. 340, eq. (3.351.2)], we can express (A.20) as

$$
\begin{gathered}
F_{\gamma}(\gamma)=1-\left[\sum_{i=1}^{4} \sum_{h=1}^{m} \sum_{t=0}^{\infty} \frac{A_{i, h}}{\bar{\gamma}(t !)^{2}}\left(C_{1 i} \frac{C_{3 i}^{t}}{\bar{\gamma}^{t}}+C_{2 i} \frac{C_{4 i}^{t}}{\bar{\gamma}^{t}}\right)\right. \\
\left.\times\left(\frac{\bar{\gamma}}{B_{h}}\right)^{t+1} \Gamma\left(t+1, B_{h} \frac{\gamma}{\bar{\gamma}}\right)\right]
\end{gathered}
$$

$$
\begin{gathered}
=1-\left[\sum_{i=1}^{4} \sum_{h=1}^{m} \sum_{t=0}^{\infty} \frac{A_{i, h} \Gamma\left(t+1, B_{h} \gamma / \bar{\gamma}\right)}{(t !)^{2} B_{h}^{t+1}}\right. \\
\left.\times\left(C_{1 i} C_{3 i}^{t}+C_{2 i} C_{4 i}^{t}\right)\right]
\end{gathered}
$$

Following the same steps as above, we can arrive at the approximate form of $F_{\gamma}(\gamma)$ as

$$
\begin{aligned}
F_{\gamma}(\gamma) \approx 1-\left[\sum_{i=1}^{4} \sum_{h=1}^{m}\right. & \sum_{t=0}^{t_{\max }} \frac{A_{i, h} \Gamma\left(t+1, B_{h} \gamma / \bar{\gamma}\right)}{(t !)^{2} B_{h}^{t+1}} \\
\times & \left.\left(D_{1 t} C_{1 i} C_{3 i}^{t}+D_{2 t} C_{2 i} C_{4 i}^{t}\right)\right] .
\end{aligned}
$$

The results obtained in (A.22) and (A.23) are put back in (7) to obtain the final expressions in Proposition 1.

\section{APPENDIX B}

\section{PROOF OF PROPOSITION 2}

Starting from (8) and putting back the first line of (7) back in (8), we can rewrite (8) as

$$
\begin{aligned}
P_{b}^{\text {Binary }}(e)= & \frac{\alpha^{\beta}}{2 \Gamma(\beta)} \int_{0}^{\infty} \gamma^{\beta-1} e^{-\alpha \gamma}\left[1-\left[\sum_{i=1}^{4} \sum_{h=1}^{m} \sum_{t=0}^{\infty} \frac{A_{i, h}}{(t !)^{2}}\right.\right. \\
& \left.\left.\times \frac{\Gamma\left(t+1, B_{h} \gamma / \bar{\gamma}\right)}{B_{h}^{t+1}}\left(C_{1 i} C_{3 i}^{t}+C_{2 i} C_{4 i}^{t}\right)\right]\right] \mathrm{d} \gamma
\end{aligned}
$$

Using the integral solution from[17, p. 340, eq. (3.351.3)], we can express (B.24) as

$$
\begin{aligned}
P_{b}^{\text {Binary }}(e)= & \frac{1}{2}-\left[\sum_{i=1}^{4} \sum_{h=1}^{m} \sum_{t=0}^{\infty} \frac{A_{i, h}}{(t !)^{2} B_{h}^{t+1}}\left(C_{1 i} C_{3 i}^{t}+C_{2 i} C_{4 i}^{t}\right)\right. \\
& \left.\times \frac{\alpha^{\beta}}{2 \Gamma(\beta)} \int_{0}^{\infty} \gamma^{\beta-1} e^{-\alpha \gamma} \Gamma\left(t+1, B_{h} \gamma / \bar{\gamma}\right) \mathrm{d} \gamma\right] .
\end{aligned}
$$

Using the integral solution from [17, eq. 6.455.1, p. 657], we can obtain

$$
\begin{aligned}
P_{b}^{\text {Binary }}(e)= & \frac{1}{2}-\left[\sum_{i=1}^{4} \sum_{h=1}^{m} \sum_{t=0}^{\infty} \frac{A_{i, h}}{(t !)^{2}}\left(C_{1 i} C_{3 i}^{t}+C_{2 i} C_{4 i}^{t}\right)\right. \\
& \times \frac{\alpha^{\beta} \bar{\gamma}^{\beta}}{2 \beta \Gamma(\beta)} \frac{\Gamma(\beta+t+1)}{\left(\alpha \bar{\gamma}+B_{h}\right)^{\beta+t+1}} \\
& \left.\times{ }_{2} F_{1}\left(1, \beta+t+1 ; \beta+1 ; \frac{\alpha \bar{\gamma}}{\alpha \bar{\gamma}+B_{h}}\right)\right] .
\end{aligned}
$$

Following the same steps as above and putting the second line of (7), we can arrive at the approximate form of $P_{b}^{\text {Binary }}(e)$ as

$$
\begin{aligned}
P_{b}^{\text {Binary }}(e) \approx & \frac{1}{2}-\left[\sum_{i=1}^{4} \sum_{h=1}^{m} \sum_{t=0}^{t_{\max }} \frac{A_{i, h}}{(t !)^{2}}\left(D_{1 t} C_{1 i} C_{3 i}^{t}+D_{2 t} C_{2 i} C_{4 i}^{t}\right)\right. \\
& \times \frac{\alpha^{\beta} \bar{\gamma}^{\beta}}{2 \beta \Gamma(\beta)} \frac{\Gamma(\beta+t+1)}{\left(\alpha \bar{\gamma}+B_{h}\right)^{\beta+t+1}} \\
& \left.\times{ }_{2} F_{1}\left(1, \beta+t+1 ; \beta+1 ; \frac{\alpha \bar{\gamma}}{\alpha \bar{\gamma}+B_{h}}\right)\right] .
\end{aligned}
$$

651 
The results obtained in (B.26) and (B.27) are put back in (9) to obtain the final expressions in Proposition 2.

\section{APPENDIX C}

\section{Proof of Proposition 3}

Starting from (10) and putting back the first line of (7) back in (10), we can rewrite (10) as

$$
\begin{aligned}
P_{b}^{M-\text { ary }}(e, g)= & \frac{1}{\sqrt{2}} \int_{0}^{\infty} e^{-v^{2} / 2}\left[1-\left[\sum_{i=1}^{4} \sum_{h=1}^{m} \sum_{t=0}^{\infty} \frac{A_{i, h}}{(t !)^{2}}\right.\right. \\
& \left.\left.\times \frac{\Gamma\left(t+1, B_{h} v^{2} / g \bar{\gamma}\right)}{B_{h}^{t+1}}\left(C_{1 i} C_{3 i}^{t}+C_{2 i} C_{4 i}^{t}\right)\right]\right] \mathrm{d} \gamma .
\end{aligned}
$$

Using the integral solution from [17, p.340, eq. (3.351)], we can express (C.28) as

$$
\begin{aligned}
P_{b}^{M-\operatorname{ary}}(e, g)= & \frac{1}{2}-\left[\sum _ { i = 1 } ^ { 4 } \sum _ { h = 1 } ^ { m } \sum _ { t = 0 } ^ { \infty } \frac { A _ { i , h } } { ( t ! ) ^ { 2 } B _ { h } ^ { t + 1 } } \left(C_{1 i} C_{3 i}^{t}+C_{2 i}\right.\right. \\
& \left.\left.\times C_{4 i}^{t}\right) \frac{1}{\sqrt{2}} \int_{0}^{\infty} e^{-v^{2} / 2} \Gamma\left(t+1, B_{h} v^{2} / g \bar{\gamma}\right) \mathrm{d} \gamma\right] .
\end{aligned}
$$

Putting $v^{2}=r$ with change of variables and using the integral solution from [17, p. 657, eq. (6.455.1)], we can obtain

$$
\begin{aligned}
& P_{b}^{M \text {-ary }}(e, g) \\
& =\frac{1}{2}-\left[\sum_{i=1}^{4} \sum_{h=1}^{m} \sum_{t=0}^{\infty} \frac{A_{i, h}}{(t !)^{2}}\left(C_{1 i} C_{3 i}^{t}+C_{2 i} C_{4 i}^{t}\right)\right. \\
& \left.\quad \times \frac{2^{t+1} \sqrt{g \bar{\gamma}} \Gamma(t+3 / 2)}{\left(g \bar{\gamma}+2 B_{h}\right)^{t+3 / 2}}{ }_{2} F_{1}\left(1, t+\frac{3}{2} ; \frac{3}{2} ; \frac{g \bar{\gamma}}{g \bar{\gamma}+2 B_{h}}\right)\right] .
\end{aligned}
$$

Following the same steps as above and putting the second line of (7), we can arrive at the approximate form of $P_{b}^{M \text {-ary }}(e, g)$ as

$$
P_{b}^{M \text {-ary }}(e, g)
$$$$
\approx \frac{1}{2}-\left[\sum_{i=1}^{4} \sum_{h=1}^{m} \sum_{t=0}^{t_{\max }}\left(D_{1 t} C_{1 i} C_{3 i}^{t}+D_{2 t} C_{2 i} C_{4 i}^{t}\right)\right.
$$

$$
\left.\times \frac{A_{i, h}}{(t !)^{2}} \frac{2^{t+1} \sqrt{g \bar{\gamma}} \Gamma(t+3 / 2)}{\left(g \bar{\gamma}+2 B_{h}\right)^{t+3 / 2}}{ }_{2} F_{1}\left(1, t+\frac{3}{2} ; \frac{3}{2} ; \frac{g \bar{\gamma}}{g \bar{\gamma}+2 B_{h}}\right)\right] .
$$

The results obtained in (C.30) and (C.31) are put back in (11) to obtain the final expressions in Proposition 3.

\section{REFERENCES}

[1] H. Suzuki, "A statistical model for urban radio propogation," IEEE Trans. Commun., vol. COM-25, no. 7, pp. 673-680, Jul. 1977.

[2] T. T. Tjhung and C. C. Chai, "Fade statistics in Nakagami-lognormal channels," IEEE Trans. Commun., vol. 47, no. 12, pp. 1769-1772, Dec. 1999.

[3] P. S. Bithas, N. C. Sagias, P. T. Mathiopoulos, G. K. Karagiannidis, and A. A. Rontogiannis, "On the performance analysis of digital communications over generalized- $K$ fading channels," IEEE Commun. Lett., vol. 10, no. 5, pp. 353-355, May 2006.
[4] I. Dey, G. G. Messier, and S. Magierowski, "Joint fading and shadowing model for large office indoor WLAN environments," IEEE Trans. Antennas Propag., vol. 62, no. 4, pp. 2209-2222, Apr. 2014.

[5] G. D. Durgin, T. S. Rappaport, and D. A. D. Wolf, "New analytical models and probability density functions for fading in wireless communications," IEEE Trans. Commun., vol. 50, no. 6, pp. 1005-1015, Jun. 2002.

[6] I. Dey, G. G. Messier, and S. Magierowski, "Fading statistics for the joint fading and two path shadowing channel," IEEE Wireless Commun. Lett., vol. 3, no. 3, pp. 301-304, Jun. 2014.

[7] I. Dey, G. G. Messier, and S. Magierowski, "The cumulative distribution function for the joint fading and two path shadowing channel: Expression and application," in Proc. IEEE VTC-Fall, Sep. 2014, pp. 1-5.

[8] I. Dey, G. G. Messier, and S. Magierowski, "On the capacity of joint fading and two-path shadowing channels," IEEE Trans. Veh. Technol., vol. 65, no. 1, pp. 403-408, Jan. 2016.

[9] G. P. Efthymoglou, N. Y. Ermolova, and V. A. Aalo, "Channel capacity and average error rates in generalised- $K$ fading channels," IET Commun., vol. 4, no. 11, pp. 1364-1372, Jul. 2010.

[10] I. Dey, T. A. Tsiftsis, and C. Rowell, "Achievable channel cutoff rate and bandwidth efficiency in indoor wireless environments," IEEE Trans. Veh. Technol., vol. 65, no. 12, pp. 10074-10079, Dec. 2016.

[11] W. T. Webb, L. Hanzo, and R. Steele, "Bandwidth efficient QAM schemes for Rayleigh fading channels," IEE Proc. I-Commun., Speech Vis., vol. 138, no. 3, pp. 169-175, Jun. 1991.

[12] M. Abramowitz and I. A. Stegun, Handbook of Mathematical Functions: With Formulas, Graphs, and Mathematical Tables, 9th ed. New York, NY, USA: Dover, 1972.

[13] M. K. Simon and M.-S. Alouini, Digital Communication Over Fading Channels, 2nd ed. Hoboken, NJ, USA: Wiley, 2005.

[14] Y. Zhao, P. Adve, and T. J. Lim, "Symbol error rate of selection amplifyand-forward relay systems," IEEE Commun. Lett., vol. 10, no. 11, pp. 757-759, Nov. 2006.

[15] A. Abdi and M. Kaveh, "Comparison of DPSK and MSK bit error rates for $\mathrm{K}$ and Rayleigh-lognormal fading distributions," IEEE Commun. Lett., vol. 4, no. 4, pp. 122-124, Apr. 2000

[16] R. Steele and L. Hanzo, Mobile Radio Communications: Second and Third-Generation Cellular and WATM Systems, 2nd ed. New York, NY, USA: Wiley, 1999.

[17] I. S. Gradshteyn and I. M. Ryzhik, Table of Integrals, Series, and Products, 7th ed. New York, NY, USA: Elsevier, 2007.

[18] C. Schlegel and D. J. Costello, Jr., "Bandwidth efficient coding for fading channels: Code construction and performance analysis," IEEE J. Sel. Areas Commun., vol. 7, no. 9, pp. 1356-1368, Dec. 1989.

[19] D. Divsalar and M. Simon, "Trellis coded modulation for 4800-9600 bits/s transmission over a fading mobile satellite channel," IEEE J. Sel. Areas Commun., vol. SAC-5, no. 2, pp. 162-177, Feb. 1987.

[20] I. Dey, G. G. Messier, and S. Magierowski, "Performance analysis of BPSK over joint fading and two-path shadowing channels," in Proc. IEEE VTC-Fall, Sep. 2014, pp. 1-5.

[21] W. T. Webb and R. Steele, "Variable rate QAM for mobile radio," IEEE Trans. Commun., vol. COM-43, no. 7, pp. 2223-2230, Jul. 1995.

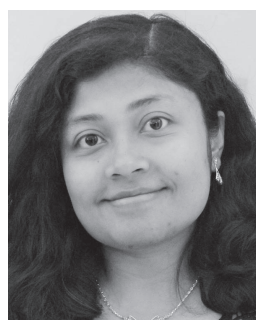

Indrakshi Dey received the B.Tech. degree (Hons.) in electronics and communication engineering from the West Bengal University of Technology, Kolkata, India, in 2005, the M.Sc. degree in wireless communications from the University of Southampton, Southampton, U.K., in 2010, and the Ph.D. degree in electrical engineering from the University of Calgary, Calgary, Canada, in 2015. From 2015 to 2016, she was a Post-Doctoral Research Fellow with the Ultra-Maritime Digital Communication Center, Dalhousie University, Halifax, Canada. She was a Research Fellow with the Department of Electronics and Telecommunications, Norwegian University of Science and Technology, Trondheim, Norway, from 2016 to 2017. Her current research interests include channel modeling, channel estimation and prediction, adaptive modulation, and dirty tape coding for different wireless propagation environments, and wireless sensor networks. In 2016, she received the prestigious research fellowship from the European Research Consortium for Information and Mathematics. 


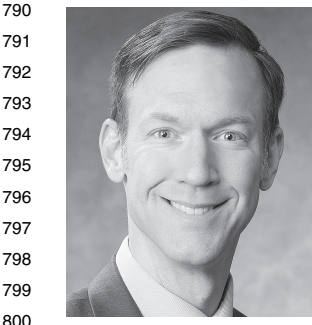

Geoffrey G. Messier received the B.S. degree (Hons.) in electrical engineering and the B.S. degree (Hons.) in computer science from the University of Saskatchewan, Canada, in 1996, the M.Sc. degree in electrical engineering from the University of Calgary, Canada in 1998, and the Ph.D. degree in electrical and computer engineering from the University of Alberta, Canada, in 2004. From 1998 to 2004, he was with the Nortel Networks CDMA Base Station Hardware Systems Design Group, Calgary, Canada. At Nortel Networks, he was responsible for radio channel propagation measurements and simulating the physical layer performance of high speed CDMA and multiple antenna wireless systems. He is currently an Associate Professor with the Department of Electrical and Computer Engineering, University of Calgary. His research interests include data networks, physical layer communications, and communications channel propagation measurements.

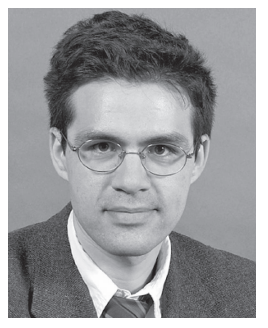

Sebastian Magierowski received the Ph.D. degree in electrical engineering from the University of Toronto in 2004. From 2004 to 2012, he served as an Assistant/Associate Professor with the Department of Electrical and Computer Engineering, University of Calgary, after which, he joined the Faculty of the Department of Electrical Engineering and Computer Science with the Lassonde School of Engineering, York University, Toronto, Canada, where he is currently an Associate Professor. As part of his industrial experience (Nortel Networks, PMC-Sierra, and Protolinx Corporation), he has involved in CMOS device modeling, high-speed mixed-signal IC design, and data networks. His research interests include analog/digital CMOS circuit design, communication systems, and biomedical instrumentation for molecular sensing and analysis.

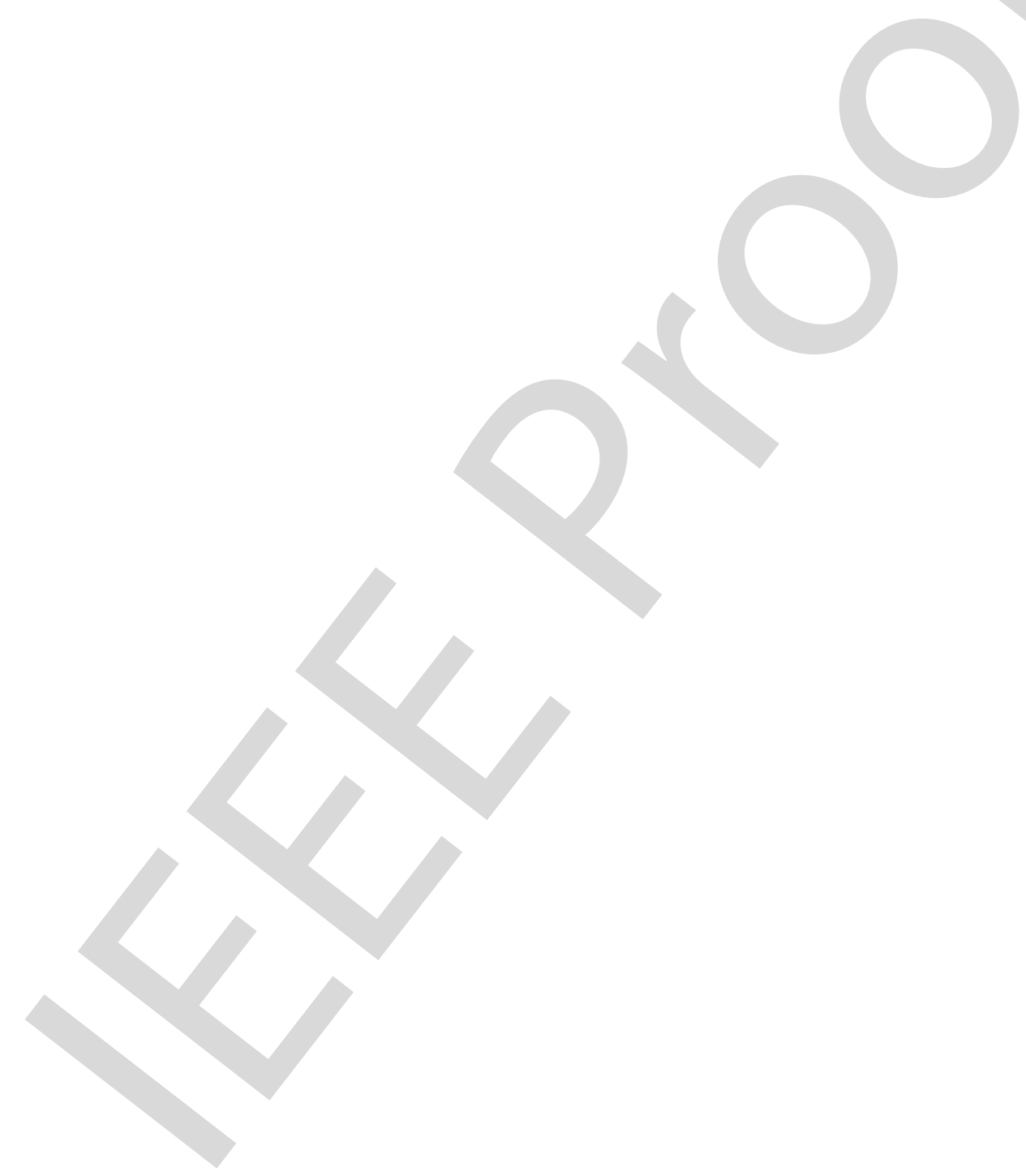

